\title{
Fibrinogen concentrate reduces intraoperative bleeding when used as first-line hemostatic therapy during major aortic replacement surgery: Results from a randomized, placebo-controlled trial
}

\author{
Niels Rahe-Meyer, MD, MSc, PhD, ${ }^{\mathrm{a}, \mathrm{b}}$ Alexander Hanke, MD, ${ }^{\mathrm{b}}$ Dirk S. Schmidt, PhD, ${ }^{\mathrm{c}}$ \\ Christian Hagl, MD, d,e and Maximilian Pichlmaier, MD $^{\mathrm{d}}$
}

\begin{abstract}
Objectives: We assessed whether fibrinogen concentrate as targeted first-line hemostatic therapy was more effective than placebo or a standardized transfusion algorithm in controlling coagulopathic bleeding in patients undergoing major aortic surgery.
\end{abstract}

\begin{abstract}
Methods: In this single-center, prospective, double-blind study, adults undergoing elective thoracic or thoracoabdominal aortic replacement surgery involving cardiopulmonary bypass were randomized to intraoperative fibrinogen concentrate $(n=29)$ or placebo $(n=32)$. Study medication was given if patients had clinically relevant coagulopathic bleeding, measured by 5 -minute bleeding mass, after cardiopulmonary bypass removal, protamine administration, and surgical hemostasis. Fibrinogen concentrate dosing was individualized using the thromboelastometric FIBTEM test. If bleeding continued, a standardized transfusion algorithm was followed. In the placebo group, all 32 patients received 1 transfusion cycle of fresh-frozen plasma/platelets, and 30 patients required a second transfusion cycle; none of these patients received any other procoagulant therapy. Change in bleeding rate after treatment was compared using $t$ tests.
\end{abstract}

Results: Mean change in bleeding rate after fibrinogen concentrate was $-48.3 \mathrm{~g} / 5 \mathrm{~min}$, compared with $0.4 \mathrm{~g} / 5$ min after placebo $(P<.001),-16.1 \mathrm{~g} / 5$ min after 1 transfusion cycle (fresh-frozen plasma or platelets; $P=.003)$, and $-28.0 \mathrm{~g} / 5 \mathrm{~min}$ after 2 transfusion cycles (fresh-frozen plasma and platelets; $P=.11$ ). Reductions in bleeding rate were greater for patients with higher bleeding rates before treatment, especially with fibrinogen concentrate.

Conclusions: FIBTEM-guided intraoperative hemostatic therapy with fibrinogen concentrate is more effective than placebo in controlling coagulopathic bleeding during major aortic replacement surgery. Fibrinogen concentrate is also more effective than 1 cycle of fresh-frozen plasma/platelets and is more rapid than —and at least as effective as - 2 cycles of fresh-frozen plasma/platelets. (J Thorac Cardiovasc Surg 2013;145:S178-85)

Complex cardiac surgery can be accompanied by excessive perioperative bleeding, which may lead to loss and

From the Clinic for Anesthesiology and Intensive Care Medicine, ${ }^{a}$ Franziskus Hospital, Bielefeld, Germany; Clinic for Anesthesiology and Intensive Care Medicine, ${ }^{b}$ Hannover Medical School, Hannover, Germany; CSL Behring, ${ }^{\mathrm{c}}$ Marburg, Germany; Clinic for Cardiothoracic, Transplant and Vascular Surgery, ${ }^{\mathrm{d}}$ Hannover Medical School, Hannover, Germany; and Cardiac Surgery Clinic, ${ }^{e}$ University Clinic Grosshadern, Munich, Germany.

Funding: This study was supported by research funding from CSL Behring. The sponsor participated in the study design with the Principal Investigator, but was not involved in data collection, data analysis and interpretation, or writing the report. The authors had full access to the data and sole authority in deciding to submit for publication. Editorial assistance was provided by Meridian HealthComms, funded by CSL Behring.

Disclosures: Drs Rahe-Meyer, Hanke, Hagl, Pichlmaier, and Schmidt received research funding from CSL Behring to perform this study. Dr Niels Rahe-Meyer has participated in advisory boards and received speaker honoraria and research support from CSL Behring and Tem International. Dr Dirk S. Schmidt is an employee of CSL Behring.

Read at the American Association for Thoracic Surgery Aortic Symposium 2012, New York, New York, April 26-27, 2012.

Received for publication May 4, 2012; revisions received Dec 19, 2012; accepted for publication Dec 28, 2012.

Address for reprints: Niels Rahe-Meyer, MD, MSc, PhD, Clinic for Anesthesiology and Intensive, Care Medicine, Franziskus Hospital, Kiskerstr. 26, D-33615 Bielefeld, Germany (E-mail: Niels.Rahe-Meyer@franziskus.de). $0022-5223 / \$ 36.00$

Copyright @ 2013 Published by Elsevier Inc. on behalf of The American Association for Thoracic Surgery

http://dx.doi.org/10.1016/j.jtcvs.2012.12.083 consumption of coagulation factors. Impairment of coagulation may be further aggravated by hypothermic circulatory arrest ${ }^{1}$ and coagulopathy associated with the use of cardiopulmonary bypass $(\mathrm{CPB})^{2,3}$ Volume replacement therapy may also be required, potentially resulting in dilutional coagulopathy. ${ }^{4,5}$ Failure to manage these coagulopathies and control blood loss can increase the risk of subsequent morbidity and mortality. ${ }^{6}$

Acquired hypofibrinogenemia is a common coagulopathy associated with cardiac surgery, and baseline plasma fibrinogen levels have been reported to decrease by $34 \%$ to $58 \%$ during $\mathrm{CPB}^{7-9}$ Because fibrinogen is the precursor to fibrin, low fibrinogen levels can compromise fibrin-based clot formation. Fibrin clot quality, recorded using the thromboelastometry-based FIBTEM test (ROTEM device; TEM International, Munich, Germany), has been shown to decrease by $38 \%$ to $52 \%$ during CPB. ${ }^{10,11}$ Time spent on CPB is therefore an important factor and typically greater for complex cardiac surgeries accompanied by substantial blood loss than for procedures such as coronary artery bypass graft surgery.

Low plasma fibrinogen levels in patients undergoing cardiac surgery are associated with increased postoperative blood loss. ${ }^{7,12-14}$ Consequently, fibrinogen replacement 


$$
\begin{aligned}
& \text { Abbreviations and Acronyms } \\
& \text { CPB = cardiopulmonary bypass } \\
& \text { FC }=\text { fibrinogen concentrate group } \\
& \text { FFP }=\text { fresh-frozen plasma } \\
& \Delta \text { rate }=\text { change in the rate of bleeding }
\end{aligned}
$$

therapy targeting a high-normal level of plasma fibrinogen and fibrin-based clot formation may be an important first step in restoring hemostasis during major bleeding after CPB. Fibrinogen replacement therapy may involve transfusion with fresh-frozen plasma (FFP) or cryoprecipitate. However, FFP has a low and variable concentration of fibrinogen ${ }^{15}$ and cannot be used when targeting a highnormal plasma fibrinogen level. ${ }^{8}$ Furthermore, the hemostatic efficacy of FFP has been questioned. ${ }^{16,17}$ Cryoprecipitate has been withdrawn from many countries because of safety concerns, ${ }^{18}$ and although it remains an option in some countries, it is not appropriate as first-line hemostatic therapy. Fibrinogen concentrate may be used for fibrinogen replacement and, unlike cryoprecipitate, undergoes purification and virus inactivation/removal steps during manufacturing. Fibrinogen concentrate is widely used for congenital fibrinogen deficiencies and reported to be potentially effective in acquired hypofibrinogenemia. ${ }^{16,19}$ Clinical evidence from prospective randomized trials is needed to demonstrate the efficacy of fibrinogen concentrate in controlling perioperative bleeding.

Our group performed a double-blind, randomized, placebo-controlled trial to investigate the efficacy of goal-directed, FIBTEM-guided fibrinogen concentrate as first-line hemostatic therapy during aortic replacement surgery. To be included in the study, patients had to exhibit clinically relevant bleeding before administration of fibrinogen concentrate or placebo, determined by measurement of the rate of coagulopathic bleeding after CPB discontinuation and reversal of heparin anticoagulation. Further measurements of the rate of bleeding were recorded after fibrinogen concentrate or placebo administration and again after cycles of additional hemostatic therapy (transfusion with FFP/platelets) for patients in the placebo group. The main aim of this investigation was to examine changes in the rate of bleeding recorded before and after administration of fibrinogen concentrate or placebo to determine whether fibrinogen concentrate is more effective than placebo in controlling coagulopathic bleeding (ie, achieves a greater reduction in the rate of bleeding). Additional analyses are presented that compare the reduction in the rate of bleeding after fibrinogen concentrate infusion with that achieved by transfusion of FFP or platelets in patients in the placebo group. Treatment with fibrinogen concentrate reduced average transfusion requirements for FFP, platelets, and red blood cells, and increased the number of patients avoiding transfusion altogether. ${ }^{20}$

\section{MATERIALS AND METHODS}

\section{Study Design, Patient Population, and Procedures}

This study was conducted at Hannover Medical School, Germany, and was approved by the local Ethics Committee and the German Regulatory Authorities; it was conducted in accordance with the Declaration of Helsinki and Good Clinical Practice. The study was assigned Local Ethics Committee reference code 4891M-mono, EudraCT trial number 2007-004612-31, and Clinicaltrials.gov identifier number NCT00701142. Patients aged 18 years or more and scheduled to undergo elective aortic replacement surgery with CPB were screened for eligibility between June 2008 and April 2010. We planned for 60 patients on the basis of a statistical power calculation for analysis of patients' transfusion requirements. ${ }^{20}$ Eighty patients were enrolled and randomized to fibrinogen concentrate (FC) or placebo groups by an unblinded pharmacist bound by a confidentiality agreement. Randomization numbers were assigned sequentially, in a 1:1 ratio, with a block size of 4 , stratified by surgery type. Sixty-one patients were then included in a prospective, randomized, placebo-controlled study after signed consent had been obtained (Figure 1). Exclusion criteria included patients with congenital or acquired coagulation disorders, previous surgery at the same site, stroke or myocardial infarction within 2 months before surgery, and use of aspirin, clopidogrel, or vitamin $\mathrm{K}$ antagonists within 2 to 5 days before surgery. Patients underwent 1 of 3 procedures: aortic valve with root/ascending aorta replacement $(\mathrm{n}=21)$, aortic valve with root/ascending aorta and aortic arch replacement $(n=22)$, or thoracoabdominal aortic replacement $(n=18)$.

\section{Intraoperative Measurement of the Rate of Bleeding}

After CPB discontinuation, neutralization of heparin using protamine, and surgical control of focal bleeding, the rate of coagulopathic bleeding was measured to assess eligibility for fibrinogen concentrate placebo administration. Patients had received no prior procoagulant therapy. The rate of bleeding was quantified by measuring 5-minute bleeding mass. Dry surgical cloths and compresses were weighed, applied to the surgical area for precisely 5 minutes, and then weighed again. Because the density of blood is approximately 1060 $\mathrm{g} / \mathrm{L},{ }^{21} 1 \mathrm{~g}$ of recorded mass represents approximately $1 \mathrm{~mL}$ of lost blood. We have previously used this approach to assess intraoperative bleeding during aortic surgery. ${ }^{8,10}$ On the basis of experiences at Hannover Medical School, bleeding in the range of 60 to $250 \mathrm{~g} / 5 \mathrm{~min}(\sim 680-2800 \mathrm{~mL} / \mathrm{h})$, where macrovascular bleeding is not evident, indicates coagulopathic bleeding of the microvasculature that cannot be surgically controlled. Patients with a rate of bleeding in this range were included in the study (Figure 1).

\section{Administration of Hemostatic Therapies and Further Assessment of Bleeding}

Measurement of bleeding and administration of hemostatic intervention were continuously alternating processes (Figure 2). In the 5 minutes after the first measurement, patients with clinically relevant bleeding were infused with fibrinogen concentrate (FC group, $n=29$ ) or an equivalent volume of $0.9 \%$ saline (placebo group, $\mathrm{n}=32$ ). Fibrinogen concentrate dosing was guided by FIBTEM maximum clot firmness results, as described previously. ${ }^{8-10}$ Medication was prepared by the unblinded pharmacist, and opaque perfusion syringes were delivered to the operating room labeled with subject and study identification numbers. The content of each syringe (either $50 \mathrm{~mL}$ of $0.9 \%$ saline as placebo or $1 \mathrm{~g}$ fibrinogen concentrate dissolved in $50 \mathrm{~mL}$ water for injection) was administered in less than 20 seconds. The median total dose was $8 \mathrm{~g}$ (minimum $3 \mathrm{~g}$, maximum $14 \mathrm{~g}$ ). Anesthesiologists and surgical staff were blinded to therapy. In the 5 minutes after infusion, the second measurement of bleeding was recorded; if less than $60 \mathrm{~g} / 5 \mathrm{~min}$, hemostasis was considered achieved, and surgery proceeded to 


\section{Enrollment}

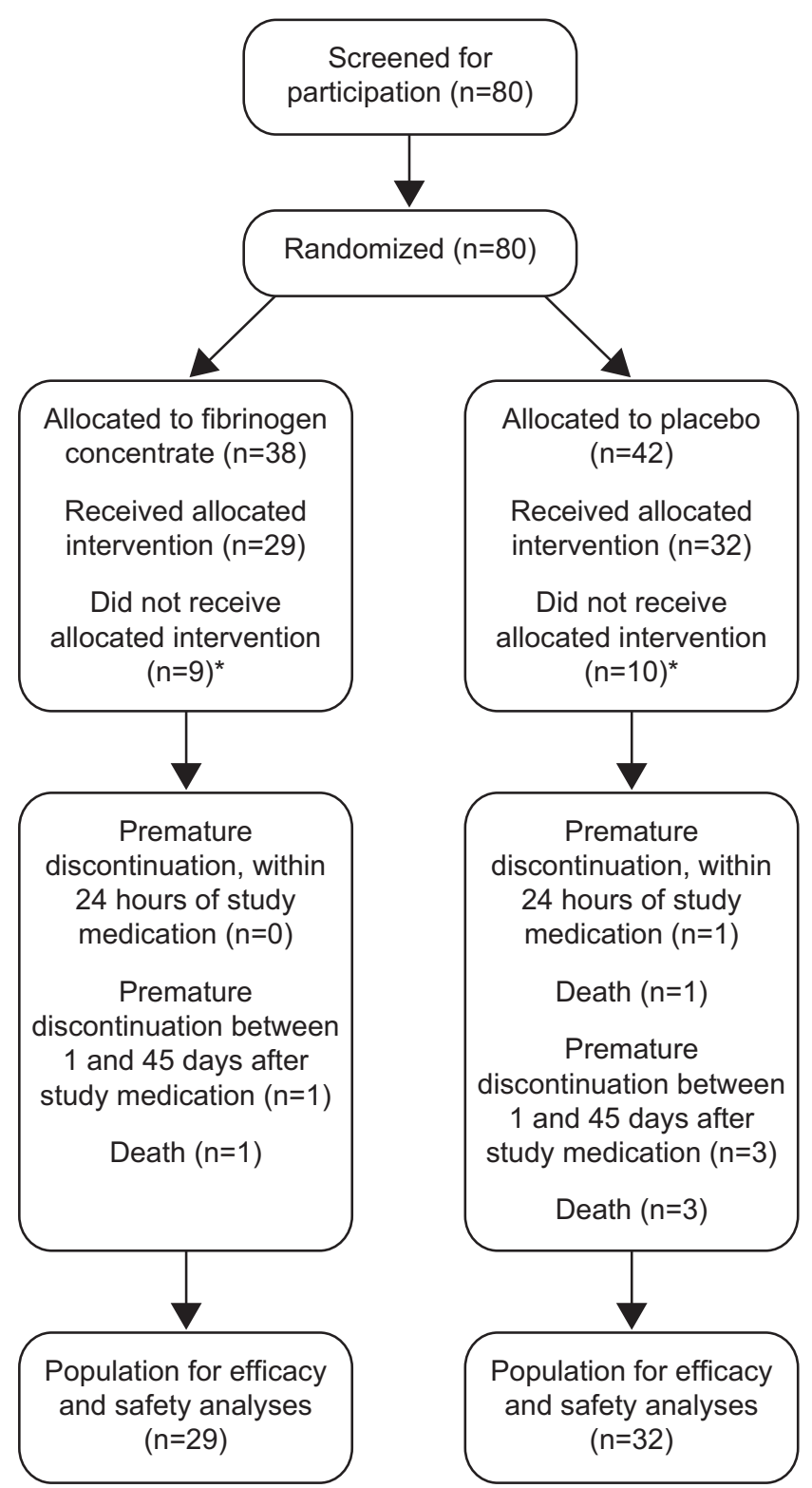

FIGURE 1. CONSORT flow diagram of patient progression through this parallel, randomized trial. *Patients did not receive the allocated intervention because the rate of bleeding was less than $60 \mathrm{~g} / 5 \mathrm{~min}$ or more than $250 \mathrm{~g} / 5 \mathrm{~min}$, FIBTEM maximum clot firmness was greater than the 22 -mm target level, or because there was a late decision not to proceed with aortic replacement surgery.

completion. When the rate of bleeding remained $60 \mathrm{~g}$ or more $/ 5 \mathrm{~min}$, patients were treated using a predefined transfusion algorithm: If platelet count at removal of the aortic clamp was less than $100,000 / \mu \mathrm{L}, 2$ units of apheresis platelet concentrate were administered; for a platelet count of $100,000 / \mu \mathrm{L}$ or more, 4 units of FFP were administered. During the 5 minutes after transfusion, the rate of bleeding was measured for a third time and a second transfusion cycle was then administered to patients still bleeding $60 \mathrm{~g}$ or more $/ 5 \mathrm{~min}$. Two units of platelets were given to patients who received 4 units FFP in the first transfusion cycle, and vice versa. The fourth measurement was recorded in the 5 minutes immediately after this second transfusion cycle, and patients with coagulopathic bleeding continued to receive further cycles of concurrent transfusion with FFP and platelets. Because of the decreasing number of patients requiring extended therapy, meaningful statistical analysis of later interventions and their subsequent effects on bleeding could not be performed. Several patients in the FC group went on to receive FFP or platelets after the second bleeding rate measurement. However, only the first and second measurements of the rate of bleeding (ie, at baseline and post-fibrinogen concentrate) are analyzed for patients in the FC group in this report, because meaningful assessment of changes in the rate of bleeding after FFP/platelet transfusions would be confounded by the initial fibrinogen concentrate administration.

\section{Statistical Analysis}

To determine the hemostatic effects of fibrinogen concentrate and placebo, the change in the rate of bleeding ( $\Delta$ rate) from the first measurement (pre-infusion) to the second measurement (post-infusion) was calculated (Figure 2) for all patients in both groups. All data were normally distributed so the $\Delta$ rate for each group is presented as mean (standard deviation). Individual $\Delta$ rate values were compared between groups using an unpaired $t$ test. The spread of $\Delta$ rate values 


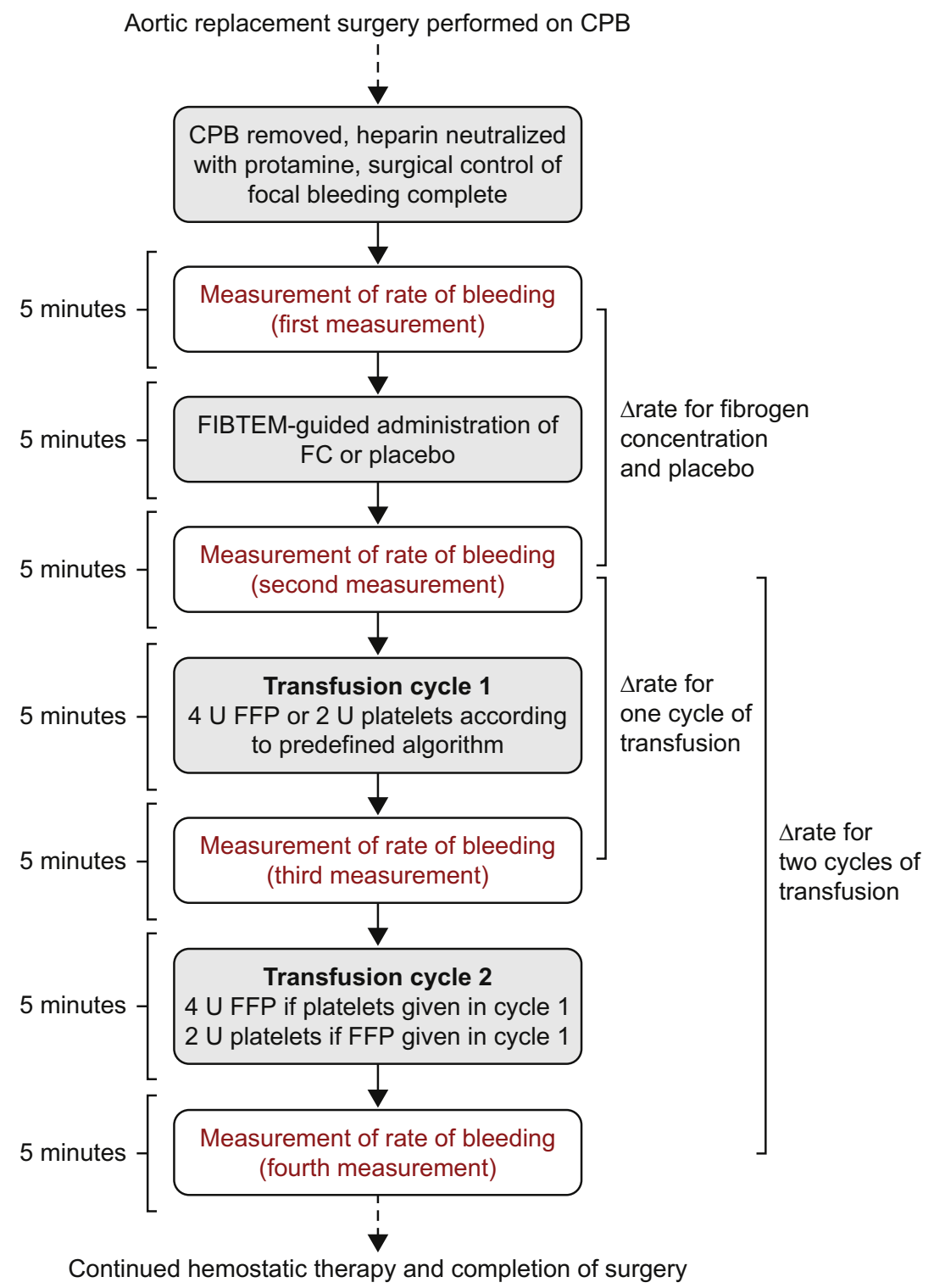

FIGURE 2. Sequence of intraoperative events illustrating the timing of measurements of the rate of bleeding and administration of hemostatic therapies, and the time-points used to calculate changes in the rate of bleeding ( $\triangle \mathrm{rate})$ after different hemostatic interventions. $C P B$, Cardiopulmonary bypass; $F C$, fibrinogen concentrate; FFP, fresh-frozen plasma; FIBTEM, fibrin-based thromboelastometry test.

was higher for the FC group than for the placebo group; to allow for this, a Welch correction was used. This analysis was a prespecified efficacy end point.

To determine the hemostatic effect of 1 cycle of transfusion ( 4 units FFP or 2 units platelets), the $\Delta$ rate from the second measurement (pre-transfusion cycle 1) to the third measurement (post-transfusion cycle 1) of the rate of bleeding was calculated. This calculation was performed using only the placebo group. By using the individual $\Delta$ rate values calculated for 1 transfusion cycle, the hemostatic effects of fibrinogen concentrate versus 1 transfusion cycle were compared as described for fibrinogen concentrate versus placebo. The hemostatic effects of 1 transfusion cycle versus placebo (a within-group comparison) were compared using a paired $t$ test.

To determine the hemostatic effect of 2 cycles of transfusion (total of 4 units FFP and 2 units platelets), the $\Delta$ rate from the second measurement (pre-transfusion cycle 1) to the fourth measurement (post-transfusion cycle 2) of the rate of bleeding was calculated. This was performed for the 30 of
32 patients in the placebo group who received 2 cycles of transfusion; no patients in the FC group were included. By using the individual $\Delta$ rate values calculated for 2 transfusion cycles, the hemostatic effects of fibrinogen concentrate versus 2 transfusion cycles were compared as described for fibrinogen concentrate versus placebo. The hemostatic effects of 2 transfusion cycles versus placebo were compared using a paired $t$ test. Trend lines on graphs represent linear regressions performed using Microsoft Excel (Microsoft Corp, Redmond, Wash).

\section{RESULTS}

\section{Patient Demographics}

Most patients were male (FC group, 66\%; placebo group, $78 \%$ ), and there were no major differences in 

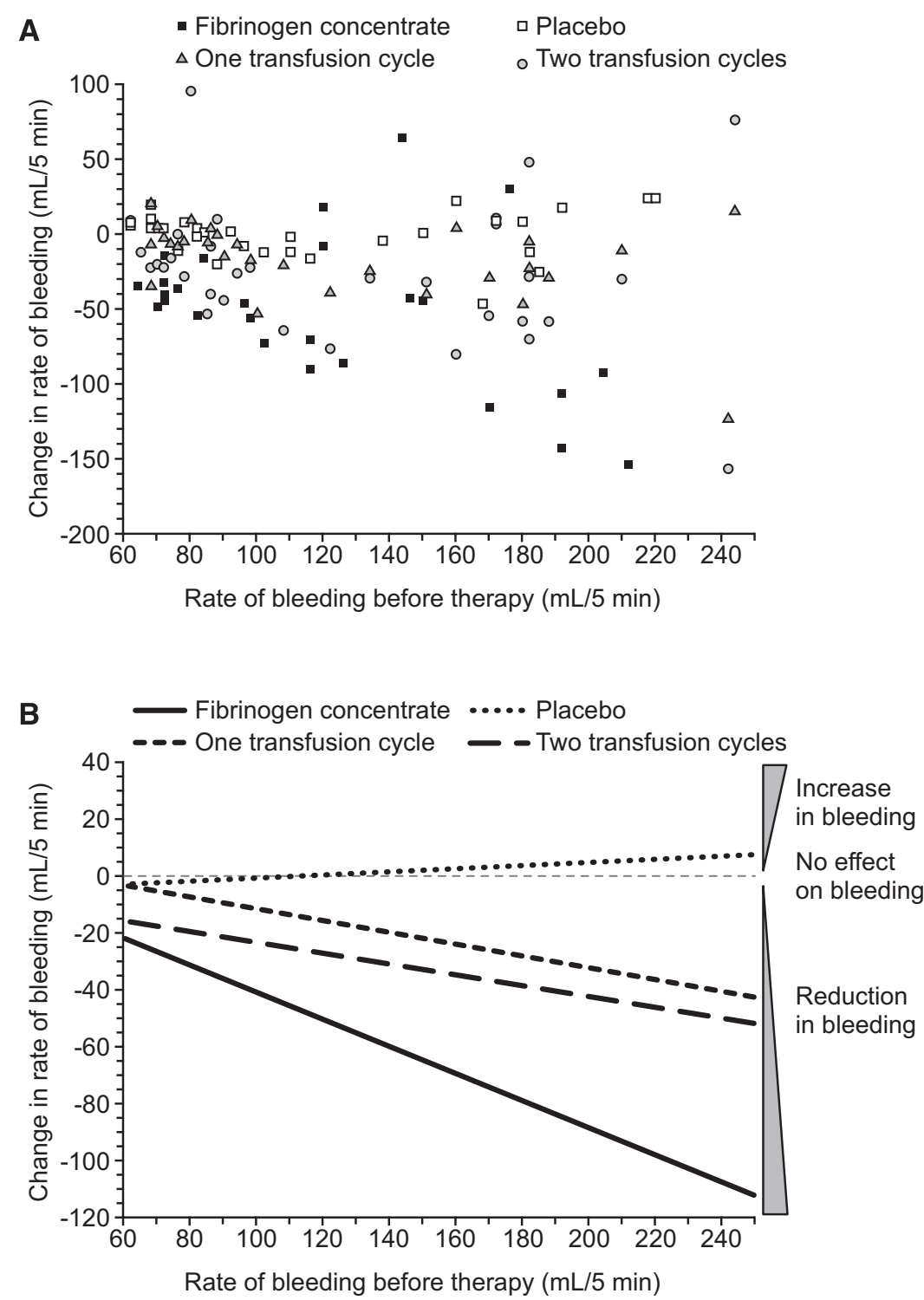

FIGURE 3. A, Individual patient data for changes in the rate of coagulopathic bleeding after treatment with placebo, 1 cycle of transfusion, 2 cycles of transfusion, or fibrinogen concentrate. B, Changing trends in the rate of bleeding after treatment with placebo, 1 cycle of transfusion, 2 cycles of transfusion, or fibrinogen concentrate. Grey dotted line, no effect.

mean age (59 vs 61 years), weight ( 88 vs $87 \mathrm{~kg}$ ), body mass index ( $\left.28 \mathrm{vs} 28 \mathrm{~kg} / \mathrm{m}^{2}\right)$, obesity ( $24 \%$ vs $22 \%$ ), preexisting medical conditions, or type of operation that would be expected to affect the results. Each group was typical for patients undergoing aortic replacement surgery.

\section{Comparing the Hemostatic Effects of Fibrinogen Concentrate Versus Placebo}

Before treatment, each group had similar mean (standard deviation) baseline rates of coagulopathic bleeding: placebo group, 121 (50) g/5 min; FC group, 116 (46) g/ 5 min. This is visible in Figure 3, A, where the values for the placebo and FC groups are comparably distributed along the $\mathrm{x}$-axis. Thus, there were no obvious differences in bleeding between the 2 groups before infusion of placebo or fibrinogen concentrate, which would be expected to cause different hemostatic responses.

Mean $\Delta$ rate was assessed post-infusion. A negative $\Delta$ rate value indicates a reduction in the rate of bleeding, whereas a positive value indicates an increase (Figure 3). Placebo treatment displayed a negligible effect on coagulopathic bleeding, with the rate of bleeding increasing by $0.4 \mathrm{~g} / 5 \mathrm{~min}(\sim 5 \mathrm{~mL} / \mathrm{h})$ (Table 1). After infusion with fibrinogen concentrate, the mean $\Delta$ rate indicated a reduction in the rate of bleeding of $48 \mathrm{~g} / 5 \mathrm{~min}$ (Table 1), approximately equivalent to $550 \mathrm{~mL} / \mathrm{h}$. More pronounced reduction in the rate of bleeding was typically observed for 
TABLE 1. Results of statistical analyses

\begin{tabular}{|c|c|c|c|c|c|c|}
\hline \multirow[b]{2}{*}{ Comparison } & \multicolumn{2}{|r|}{ Treatment A } & \multicolumn{2}{|c|}{ Treatment B } & \multirow[b]{2}{*}{ Difference mean $(95 \% \mathrm{CI})$} & \multirow[b]{2}{*}{$P$ value } \\
\hline & $\mathbf{N}$ & $\Delta$ rate mean (SD) & $\mathbf{N}$ & $\Delta$ rate mean (SD) & & \\
\hline (A) FC vs (B) placebo & 29 & $-48.3(48.6)$ & 32 & $0.4(15.1)$ & $-48.8(-67.9$ to -29.6$)$ & $<.001$ \\
\hline (A) 1 cycle of FFP/platelets vs (B) placebo & 32 & $-16.1(26.1)$ & 32 & $0.4(15.1)$ & $-16.6(-27.5$ to -5.7$)$ & .004 \\
\hline (A) 2 cycles of FFP/platelets vs (B) placebo & 30 & $-28.0(46.6)$ & 30 & $1.7(14.8)$ & $-29.7(-47.1$ to -12.3$)$ & .002 \\
\hline (A) FC vs (B) 1 cycle of FFP/platelets & 29 & $-48.3(48.6)$ & 32 & $-16.1(26.1)$ & $-32.2(-52.7$ to -11.2$)$ & .003 \\
\hline (A) FC vs (B) 2 cycles of FFP/platelets & 29 & $-48.3(48.6)$ & 30 & $-28.0(46.6)$ & $-20.3(-45.1$ to 4.6$)$ & .11 \\
\hline
\end{tabular}

Statistically significant $P$ values are in bold. $\triangle$ rate, Change in the rate of bleeding; $S D$, standard deviation; $C I$, confidence interval; $F C$, fibrinogen concentrate; $F F P$, fresh-frozen plasma.

patients exhibiting a higher rate of bleeding before fibrinogen concentrate infusion. Statistical comparison confirmed that fibrinogen concentrate was more effective than placebo for controlling intraoperative bleeding $(P<.001$, Table 1$)$.

\section{Comparing the Hemostatic Effects of 1 Cycle of Transfusion (Fresh-Frozen Plasma or Platelets) Versus Placebo}

All patients in the placebo group (32/32) received at least 1 cycle of transfusion with 4 units FFP or 2 units platelet concentrate. Because placebo infusion had little effect on the rate of bleeding, the hemostatic effect of FFP/platelet transfusion could be assessed by monitoring subsequent changes in the rate of bleeding. As a baseline for determining $\Delta$ rate after $1 \mathrm{cy}-$ cle of transfusion, the second measurement of the rate of bleeding was used (Figure 2). This value was recorded immediately before FFP/platelet transfusion, and the mean (standard deviation) rate of bleeding for the placebo group at this time was 122 (55) g/5 min, which was comparable with the rate of bleeding observed before placebo infusion. After FFP/platelets transfusion, there was an overall decrease in the rate of bleeding (Figure $3, B$ ). The mean $\Delta$ rate (Table 1) indicated that 1 transfusion cycle reduced the rate of coagulopathic bleeding by 16 $\mathrm{g} / 5 \mathrm{~min}$, equivalent to approximately $180 \mathrm{~mL} / \mathrm{h}$. This reduction was significant compared with placebo $(P=.004$, Table 1$)$.

\section{Comparing the Hemostatic Effects of 2 Cycles of Transfusion (Fresh-Frozen Plasma and Platelets) Versus Placebo}

In the placebo group, 30 of 32 patients received a second cycle of transfusion, in which patients were given whichever therapy was not received in the first cycle. Consequently, all 30 patients undergoing 2 cycles of transfusion received a total of 4 units FFP and 2 units platelets. The hemostatic effect of this combination was assessed by calculating the $\Delta$ rate between the measurements made before any transfusion (second measurement) and after 2 cycles of transfusion (fourth measurement). Transfusion with 4 units FFP plus 2 units platelets led to an overall reduction in the rate of bleeding of $28 \mathrm{~g} / 5 \mathrm{~min}$ (Figure $3, B$ ), equivalent to approximately $320 \mathrm{~mL} / \mathrm{h}$. This reduction was also significant compared with placebo $(P=.002$, Table 1$)$.

\section{Comparing the Hemostatic Effects of Fibrinogen Concentrate Versus 1 Cycle of Transfusion (Fresh- Frozen Plasma or Platelets)}

Although the first cycle of transfusion had a positive hemostatic effect, statistical comparison revealed that the reduction in the rate of bleeding observed after transfusion with FFP or platelets was significantly less than the reduction in the rate of bleeding observed after fibrinogen concentrate infusion $(P=.003$, Table 1$)$.

\section{Comparing the Hemostatic Effects of Fibrinogen Concentrate Versus 2 Cycles of Transfusion (Fresh- Frozen Plasma and Platelets)}

The decrease in the rate of bleeding after 2 cycles of transfusion $(28 \mathrm{~g} / 5 \mathrm{~min}, \sim 320 \mathrm{~mL} / \mathrm{h}$ ) was also not as great as that seen after treatment with fibrinogen concentrate (48 g/5 min, $\sim 550 \mathrm{~mL} / \mathrm{h}$ ); however, this difference did not reach statistical significance $(P=.11$, Table 1$)$.

\section{DISCUSSION}

In this single-center, prospective, randomized, doubleblind, placebo-controlled study, we used 5-minute bleeding mass to accurately quantify the rate of coagulopathic bleeding in patients undergoing major aortic replacement surgery. This unique tool has never before been used in a prospective, randomized study. We successfully used 5-minute bleeding mass as a marker of the beginning ( $\geq 60 \mathrm{~g} / 5 \mathrm{~min})$ and end ( $<60 \mathrm{~g} / 5 \mathrm{~min})$ of clinically relevant coagulopathic bleeding and of the patient's response to hemostatic therapy, avoiding the need to rely on subjective measures. Our results show that FIBTEM-guided intraoperative hemostatic monotherapy with fibrinogen concentrate is more effective than placebo, 4 units FFP, or 2 units platelets, and is at least as effective as 4 units FFP plus 2 units platelets in reducing the rate of coagulopathic bleeding during major aortic replacement surgery.

That FIBTEM-guided fibrinogen concentrate as first-line hemostatic therapy led to a significant reduction in the rate of bleeding explains why patients who received fibrinogen concentrate required fewer transfusions with allogeneic blood products than patients who received placebo. ${ }^{20}$ The difference in mean values suggests that fibrinogen concentrate may be more effective than even 2 transfusion cycles of FFP/platelets. It will be interesting to see whether this 
is confirmed in the Phase III REPLACE study (NCT01475669). Of note is that 4 units FFP plus 2 units platelets takes substantially longer to administer than 1 infusion with fibrinogen concentrate, and this is an important consideration when control of acute bleeding is urgently required. Whereas allogeneic blood products must be crossmatched and thawed, and large volumes given, fibrinogen concentrate can be stored at room temperature, rapidly reconstituted, and infused as a smaller volume. In addition, fibrinogen concentrate avoids many of the safety issues associated with allogeneic blood products, such as transfusion-related acute lung injury, transfusionassociated circulatory overload, acute respiratory distress syndrome, and transfusion-related immunomodulation. ${ }^{22-24}$

The benefits of targeted, individualized dosing of fibrinogen concentrate are evident in that changes in the rate of bleeding were more pronounced for patients with a high initial rate. This was evident to a lesser degree after transfusion with FFP/platelets, where dosing was fixed and not tailored to the individual patient. Consistent with previous studies in cardiac surgery ${ }^{8,10,25}$ and trauma, ${ }^{26}$ our findings demonstrate the benefit of using a target FIBTEM maximum clot firmness value to guide appropriate dosing of fibrinogen concentrate. This "theragnostic" approach integrates rapid diagnostic testing with drug therapy, ensuring that fibrinogen concentrate is administered and dosed in line with patients' actual needs. Two systematic reviews, which included several prospective studies in cardiovascular surgery, concluded that fibrinogen concentrate therapy could increase clot firmness and decrease transfusion requirements, blood loss, and postoperative drainage volumes. $^{16,19}$

\section{Study Limitations}

A limitation of the present analyses is that different timepoints were used as the baseline from which to measure the hemostatic effect of treatment with FFP/platelets than were used when measuring the effects of fibrinogen concentrate and placebo; however, we did ensure before performing the comparisons that the baseline values were comparable for both groups and at both time-points. We cannot rule out the possibility of selection bias in using "nonresponders" from the placebo group for the assessment of 2 transfusion cycles (FFP and platelets). However, this is expected to have had little impact, because the majority (30/ $32,94 \%$ ) of patients in the placebo group were "nonresponders" (ie, went on to receive a second cycle of transfusion). In addition, only comparisons of $\Delta$ rate after administration of fibrinogen concentrate or placebo represent comparisons between prespecified efficacy end points. Comparisons of placebo or fibrinogen concentrate versus 1 or 2 cycles of transfusion are exploratory analyses that warrant further prospective investigation. Although 5-minute bleeding mass as a tool to quantify the rate of intraoperative coagulopathic bleeding has not been used in a prospective, randomized trial, our group has successfully used it in small prospective aortic surgery studies. ${ }^{8,10}$ The present study provides further evidence that this method yields accurate and reproducible measurements.

\section{CONCLUSIONS}

A single, targeted dose of fibrinogen concentrate after discontinuation of $\mathrm{CPB}$ controls coagulopathic bleeding during aortic surgery more effectively than placebo or a standardized treatment algorithm of 4 units FFP or 2 units apheresis platelets. Fibrinogen concentrate also enables more rapid and at least as effective control of intraoperative bleeding when compared with transfusion of 4 units FFP and 2 units platelets. These findings support the use of goal-directed, FIBTEMguided fibrinogen concentrate as a rapid and efficacious first-line hemostatic therapy in patients with coagulopathic bleeding undergoing major aortic replacement surgery.

\section{References}

1. Campos JM, Paniagua P. Hypothermia during cardiac surgery. Best Pract Res Clin Anaesthesiol. 2008;22:695-709.

2. Kestin AS, Valeri CR, Khuri SF, Loscalzo J, Ellis PA, MacGregor H, et al. The platelet function defect of cardiopulmonary bypass. Blood. 1993;82:107-17.

3. Paparella D, Rotunno C, Guida P, Malvindi PG, Scrascia G, De Palo M, et al. Hemostasis alterations in patients with acute aortic dissection. Ann Thorac Surg. 2011;91:1364-9.

4. Fenger-Eriksen C, Tonnesen E, Ingerslev J, Sorensen B. Mechanisms of hydroxyethyl starch-induced dilutional coagulopathy. J Thromb Haemost. 2009;7: 1099-105.

5. Schramko A, Suojaranta-Ylinen R, Kuitunen A, Raivio P, Kukkonen S, Niemi T. Hydroxyethylstarch and gelatin solutions impair blood coagulation after cardiac surgery: a prospective randomized trial. Br J Anaesth. 2010;104:691-7.

6. Levi M, Cromheecke ME, de Jonge E, Prins MH, de Mol BJ, Briet E, et al. Pharmacological strategies to decrease excessive blood loss in cardiac surgery: a meta-analysis of clinically relevant endpoints. Lancet. 1999;354:1940-7.

7. Blome M, Isgro F, Kiessling AH, Skuras J, Haubelt H, Hellstern P, et al. Relationship between factor XIII activity, fibrinogen, haemostasis screening tests and postoperative bleeding in cardiopulmonary bypass surgery. Thromb Haemost. 2005;93:1101-7.

8. Rahe-Meyer N, Pichlmaier M, Haverich A, Solomon C, Winterhalter M, Piepenbrock S, et al. Bleeding management with fibrinogen concentrate targeting a high-normal plasma fibrinogen level: a pilot study. Br JAnaesth. 2009;102:785-92.

9. Solomon C, Pichlmaier U, Schoechl H, Hagl C, Raymondos K, Scheinichen D, et al. Recovery of fibrinogen after administration of fibrinogen concentrate to patients with severe bleeding after cardiopulmonary bypass surgery. Br J Anaesth. 2010;104:555-62.

10. Rahe-Meyer N, Solomon C, Winterhalter M, Piepenbrock S, Tanaka K, Haverich A, et al. Thromboelastometry-guided administration of fibrinogen concentrate for the treatment of excessive intraoperative bleeding in thoracoabdominal aortic aneurysm surgery. J Thorac Cardiovasc Surg. 2009;138:694-702.

11. Solomon C, Rahe-Meyer N, Sorensen B. Fibrin formation is more impaired than thrombin generation and platelets immediately following cardiac surgery. Thromb Res. 2011;128:277-82.

12. Karkouti K, McCluskey SA, Syed S, Pazaratz C, Poonawala H, Crowther MA. The influence of perioperative coagulation status on postoperative blood loss in complex cardiac surgery: a prospective observational study. Anesth Analg. 2010;110:1533-40.

13. Karlsson M, Ternstrom L, Hyllner M, Baghaei F, Nilsson S, Jeppsson A. Plasma fibrinogen level, bleeding, and transfusion after on-pump coronary artery bypass grafting surgery: a prospective observational study. Transfusion. 2008;48:2152-8.

14. Ucar HI, Oc M, Tok M, Dogan OF, Oc B, Aydin A, et al. Preoperative fibrinogen levels as a predictor of postoperative bleeding after open heart surgery. Heart Surg Forum. 2007;10:E392-6.

15. Theusinger OM, Baulig W, Seifert B, Emmert MY, Spahn DR, Asmis LM. Relative concentrations of haemostatic factors and cytokines in solvent/detergenttreated and fresh-frozen plasma. Br J Anaesth. 2011;106:505-11. 
16. Kozek-Langenecker S, Sorensen B, Hess JR, Spahn DR. Clinical effectiveness of fresh frozen plasma compared with fibrinogen concentrate: a systematic review. Crit Care. 2011;15:R239.

17. Yang L, Stanworth S, Hopewell S, Doree C, Murphy M. Is fresh-frozen plasma clinically effective? An update of a systematic review of randomized controlled trials. Transfusion. 2012;52:1673-86; quiz 1673 .

18. Sorensen B, Bevan D. A critical evaluation of cryoprecipitate for replacement of fibrinogen. Br J Haematol. 2010;149:834-43.

19. Warmuth M, Mad P, Wild C. Systematic review of the efficacy and safety of fibrinogen concentrate substitution in adults. Acta Anaesthesiol Scand. 2012;56:539-48.

20. Rahe-Meyer N, Solomon C, Hanke A, Schmidt D, Knoerzer D, Hochleitner G, et al. Effects of fibrinogen concentrate as first-line therapy during major aortic replacement surgery: a randomized, placebo-controlled trial. Anesthesiology. 2013;118:40-50.

21. Cutnell J, Johnson K. Physics, Fourth Edition. Canada: John Wiley \& Sons; 1998.
22. Dara SI, Rana R, Afessa B, Moore SB, Gajic O. Fresh frozen plasma transfusion in critically ill medical patients with coagulopathy. Crit Care Med. 2005;33:2667-71

23. Khan H, Belsher J, Yilmaz M, Afessa B, Winters JL, Moore SB, et al. Fresh-frozen plasma and platelet transfusions are associated with development of acute lung injury in critically ill medical patients. Chest. 2007;131:1308-14.

24. Knowles S, Cohen H, on behalf of the Serious Hazards of Transfusion (SHOT) Steering Group. The 2010 Annual SHOT Report (2011). Available at: http://www.shotuk.org/wp-content/uploads/2011/07/SHOT-2010-Report.pdf. Accessed March 3, 2012.

25. Cui Y, Hei F, Long C, Feng Z, Zhao J, Yan F, et al. Perioperative monitoring of thromboelastograph on blood protection and recovery for severely cyanotic patients undergoing complex cardiac surgery. Artif Organs. 2010;34:955-60.

26. Schochl H, Nienaber U, Maegele M, Hochleitner G, Primavesi F, Steitz B, et al Transfusion in trauma: thromboelastometry-guided coagulation factor concentrate-based therapy versus standard fresh frozen plasma-based therapy. Crit Care. 2011;15:R83. 